\title{
A new Approach for Obtaining Optimal Solution of Unbalanced Fuzzy Transportation Problem
}

\author{
Nidhi Joshi ${ }^{1}$, Surjeet Singh Chauhan (Gonder $)^{2}$ \\ PTU, Jalandhar, Research Scholar, College: CGC, Mohali, Punjab, India \\ nids_22@yahoo.co.in \\ Department of Applied Science, Chandigarh Group of Colleges, Gharuan, Mohali, Punjab, India \\ surjeetschauhan@yahoo.com
}

\begin{abstract}
The present paper attempts to study the unbalanced fuzzy transportation problem so as to minimize the transportation cost of products when supply, demand and cost of the products are represented by fuzzy numbers. In this paper, authors use Roubast ranking technique to transform trapezoidal fuzzy numbers to crisp numbers and propose a new algorithm to find the fuzzy optimal solution of unbalanced fuzzy transportation problem. The proposed algorithm is more efficient than other existing algorithms like simple VAM and is illustrated via numerical example. Also, a comparison between the results of the new algorithm and the result of algorithm using simple VAM is provided.
\end{abstract}

\section{Indexing terms/Keywords}

Optimization; Unbalanced Fuzzy transportation problem; Roubast Ranking technique; Trapezoidal fuzzy number; optimal solution; Modified VAM; Total opportunity cost.

\section{Academic Discipline And Sub-Disciplines}

Mathematics and Fuzzy transportation problem

\section{MATHEMATICS SUBJECT CLASSIFICATION}

90C08, 90C90

\section{TYPE (METHOD/APPROACH)}

Illustration of proposed algorithm using numerical example.

\section{INTRODUCTION}

In the conventional transportation problems it is assumed that decision maker is sure about the precise values of transportation cost, availability and demand of the product. However, in real world applications, all the parameters of the transportation problems may not be known precisely due to uncontrollable factors. This type of imprecise data may not always be well represented by random variables selected from a probability distribution. Fuzzy numbers introduced by Zadeh[1] in 1965, may represent this kind of data. Method of fuzzy decision making, therefore, is needed here. Fuzzy transportation problem (FTP) is the problem of minimizing fuzzy valued objective functions with fuzzy source and fuzzy destination parameters. The balanced condition is both a necessary and sufficient condition for the existence of a feasible solution to the transportation problem. In this paper, the authors attempt to study the optimal solution of Unbalanced Fuzzy Transportation Problem using Modified VAM with Roubast Ranking Technique. In 1996, Chanas and Kuchta[2] introduced the concept of the optimal solution for the transportation problem with fuzzy coefficients expressed as fuzzy numbers and developed an algorithm for obtaining the optimal solution. Saad and Abbas [4], in 2003, discussed the solution algorithm for solving the transportation problem in fuzzy environment. Their method aims to obtain positive optimal fuzzy transportation cost in most cases. Liu and Kao [3], in 2004, described a method for solving fuzzy transportation problems based on extension principle. The study concluded that the membership function of the objective value of the equality problem is contained in that of the inequality problem. In 2006, Gani and Razak[5] presented a two stage cost minimizing fuzzy transportation problem in which supplies and demands i.e. transportation cost, availability and demand of the product are trapezoidal fuzzy numbers. In 2011, Amit kumar and Amarpreet kaur[19] proposed a new method for solving unbalanced fuzzy transportation problem using the ranking function where transportation cost, availability and demand of product are represented by generalized fuzzy numbers. Amit Kumar and Amarpreet kaur[17] in 2011, proposed an application of linear programming for solving unbalanced fuzzy transportation problem. In 2012, Amit kumar and Amarpreet kaur[18] proposed two new methods based on fuzzy linear programming formulation and classical transportation method to obtain the optimal solution of unbalanced fuzzy transportation problem, in their paper they showed that the results obtained by both the methods were same. A. Edward Samuel amd M. Venkatachalapathy[15], in 2012, proposed a new dual based approach for unbalanced fuzzy transportation problem, in which they used the linear programming duality theory to check the optimal condition and to obtain the optimal solution of the problem. In $2013 \mathrm{~K}$. Kadhirvel and K. Balamurugan[14] solved the unbalanced transportation problem using simple VAM and then the optimal solution is obtained by fuzzy U-V method similar to already existing MODI method. In 2013, A. Edward Samuel and M. Venkatachalapathy[13], proposed an improved zero point method (IZPM) for solving the unbalanced fuzzy transportation problem using triangular fuzzy numbers and concluded that the result obtained by IZPM is similar to that obtained by MODI method. In 2014, Deepika Rani et al.[12] proposed a new linear programming approach to solve the unbalanced fuzzy transportation problem. Also, in 2014, K.R.Sobha[16], discussed the profit maximisation of unbalanced fuzzy transportation problem. In her paper, she used Yager's ranking technique for transforming triangular fuzzy numbers to 


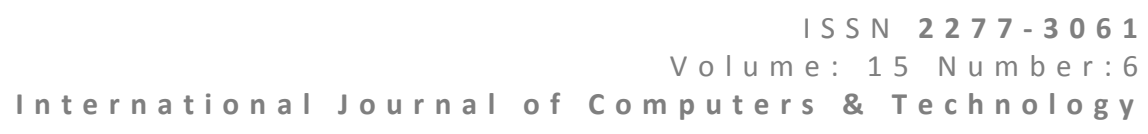

crisp numbers in conjunction with the simple VAM to obtain optimal solution. In 2015, R.K. Saini et al.[11] solved the unbalanced fuzzy transportation problem using centroid ranking technique in conjunction with zero suffix method.

In this paper, authors attempt to solve the unbalanced transportation problem using a new algorithm based on the practical approach. In order to solve the unbalanced transportation problem, we first need to change it into a balanced transportation problem. Historically, authors generally use either a dummy row or a dummy column to balance the unbalanced problem. However, there is no existence of dummy in reality, the excess availability is not transported at all is held back at one or more origins. In the present paper authors provide additional information that to which of the destination(s) the excess availability is to be transported for future demand at minimum cost.

Mathematically a transportation problem can be stated as follows:

minimize

$$
\mathrm{Z}=\sum_{i=1}^{m} \sum_{j=1}^{n} c_{i j} x_{i j}
$$

subject to

$$
\begin{array}{r}
\sum_{j=1}^{n} x_{i j}=\mathrm{a}_{i} \quad i=1,2, \ldots \ldots \ldots \ldots, m \\
\sum_{i=1}^{m} x_{i j}=b_{j} \quad j=1,2, \ldots \ldots \ldots \ldots, n \\
\mathrm{x}_{i j} \geq 0 \quad i=1,2, \ldots \ldots \ldots \ldots \ldots, m \quad j=1,2, \ldots \ldots \ldots \ldots \ldots \ldots \ldots \ldots \ldots \ldots
\end{array}
$$

where $\mathbf{c}_{i j}$ is the cost of transportation of an unit from the $i^{\text {th }}$ source to the $j^{\text {th }}$ destination, and the quantity $\boldsymbol{x}_{i j}$ is to be some positive integer or zero, which is to be transported from the ith origin to jth destination. An obvious necessary and sufficient condition for the linear programming problem given in (1) to have a solution is that

$$
\sum_{i=1}^{m} a_{i}=\sum_{j=1}^{n} b_{j}
$$

i.e. assume that total availability is equal to the total requirement. If it is not true, then the unbalanced problem should be balanced by adding a dummy source with availability equal to total availability as well as a dummy destination with demand equal to sum of total availability and excess supply. It should be noted that the problem has a feasible solution if and only if the condition (2) satisfied. Now, the problem is to determine $\boldsymbol{x}_{\boldsymbol{i}}$, in such a way that the total transportation cost is minimum.

In fuzzy environment a transportation problem can be stated as follows:

minimize

$$
\mathrm{Z}=\sum_{i=1}^{m} \sum_{j=1}^{n} \tilde{c}_{i j} \tilde{X}_{i j}
$$

Subject to

$$
\begin{array}{cr}
\sum_{j=1}^{n} \tilde{x}_{i j}=\tilde{a}_{i} \quad & i=1,2 \ldots \ldots \ldots \ldots m \\
\sum_{i=1}^{m} \tilde{x}_{i j}=\tilde{b}_{j} & j=1,2 \ldots \ldots \ldots \ldots n \\
x i j \geq 0 \quad i=1,2, \ldots \ldots \ldots, m & j=1,2, \ldots \ldots \ldots
\end{array}
$$
, n

in which the transportation costs $\tilde{c}_{i j}$, supply $\tilde{a}_{i}$ and demand $\tilde{b}_{j}$ quantities are fuzzy quantities. An obvious necessary and sufficient condition for the fuzzy transportation problem given in (4-5) to have a solution is that

$$
\sum_{i=1}^{m} \tilde{a}_{i} \cong \sum_{j=1}^{n} \tilde{b}_{j}
$$

i.e. the total fuzzy availability should be equal to the total fuzzy requirement. If it is not true, then the unbalanced problem should be balanced by adding a dummy source with availability equal to total fuzzy availability as well as a dummy destination with demand equal to sum of total fuzzy availability and excess supply. It should be noted that the problem has a feasible solution if and only if the condition (5) satisfied.

The present paper is organized as follows: In section 2 few basic definitions, Roubast ranking Technique, Trapezoidal fuzzy numbers and their arithmetic operations are reviewed. Proposed algorithm for Modified Vogel Approximation Method and the traditional algorithm using Simple VAM are illustrated for comparative study in section 3. Next, in Section 4 both algorithms are illustrated with the help of a Numerical example. Section 5 is the conclusion section and section 6 highlights the advantages of proposed algorithm. 


\section{PRELIMINARIES}

\section{Fuzzy Set}

A fuzzy set is characterized by a membership function mapping element of a domain, space or universe of discourse $X$ to the unit interval $[0,1]$ i.e. $\tilde{A}=\left\{\left(\mathrm{x}, \mu_{\tilde{A}}(\mathrm{x}) ; \mathrm{x} \in \mathrm{X}\right\}\right.$, here

$$
\mu_{\widetilde{A}}(\mathrm{x}): \mathrm{X} \rightarrow[0,1]
$$

is a mapping called the degree of membership function of the fuzzy set $\mathrm{A}$ and $\mu_{\bar{A}}(\mathrm{x})$ is called the membership value of $\mathrm{x} \varepsilon$ $X$ in the fuzzy set $A$. These membership grades are often represented by real numbers ranging from $[0,1]$.

\section{Trapezoidal Fuzzy Numbers}

A fuzzy number $\tilde{A}=(m, n, p, q)$ is said to be trapezoidal fuzzy number if its membership function is given by

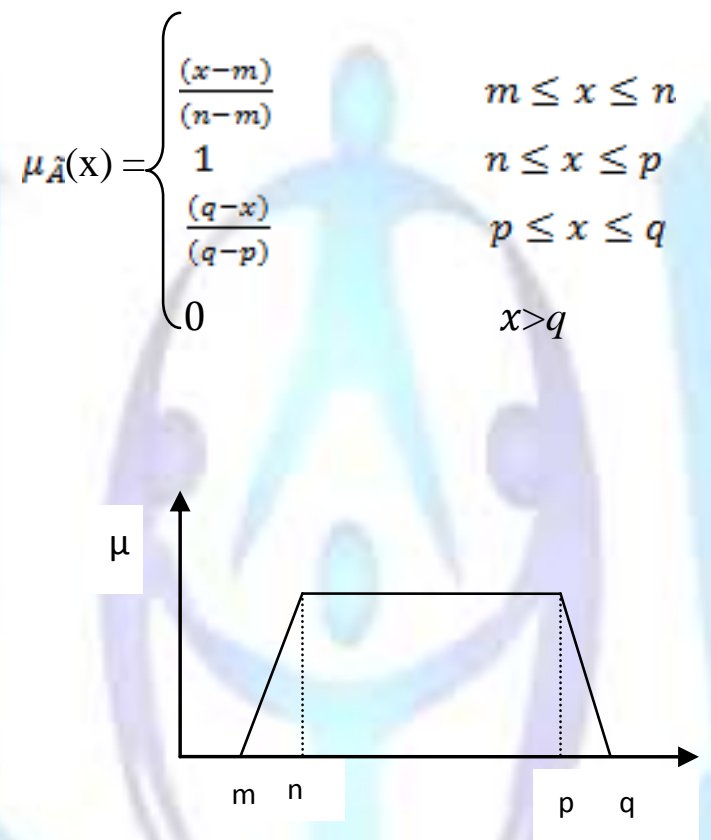

Fig 1: Trapezoidal Fuzzy Numbers

\section{Properties of Trapezoidal Fuzzy Number}

i. Trapezoidal fuzzy number $\tilde{A}=(m, n, p, q)$ is said to be non negative trapezoidal fuzzy number Iff $m-p \geq 0$

ii. A trapezoidal fuzzy number $\tilde{A}=(m, n, p, q)$ is said to be zero trapezoidal fuzzy number Iff $m=0, n=0, p=0, q=0$.

iii. Two trapezoidal fuzzy numbers $\tilde{A}_{1}=\left(m_{1}, n_{1}, p_{1}, q_{1}\right)$ and $\tilde{A}_{2}=\left(m_{2}, n_{2}, p_{2}, q_{2}\right)$ are said be equal i.e. $\tilde{A}_{1}=\tilde{A}_{2}$. Iff $m_{1}=$ $m_{2}, n_{1}=n_{2}, p_{1}=p_{2}, q_{1}=q_{2}$.

\section{Arithmetic Operators for Solving Trapezoidal Fuzzy Number}

If $\tilde{A}_{1}=\left(m_{1}, n_{1}, p_{1}, q_{1}\right)$ and $\tilde{A}_{2}=\left(m_{2}, n_{2}, p_{2}, q_{2}\right)$ two trapezoidal fuzzy numbers then the arithmetic operations on $\tilde{A}_{1}$ and $\tilde{A}_{2}$ as follows:

Addition $\tilde{A}_{1}+\tilde{A}_{2}=\left(m_{1}+m_{2}, n_{1}+n_{2}, p_{1}+p_{2}, q_{1}+q_{2}\right)$

Subtraction $\tilde{A}_{1-} \tilde{A}_{2}=\left(m_{1}-q_{2}, n_{1}-p_{2}, p_{1}-n_{2}, q_{1}-m_{2}\right)$

Multiplication $\tilde{A}_{1} \cdot \tilde{A}_{2}=\left(t_{1}, t_{2}, t_{3}, t_{4}\right)$

Where

$$
\begin{aligned}
& t_{1}=\operatorname{minimum}\left\{m_{1} m_{2}, m_{1} q_{2}, q_{1} m_{2}, q_{1} q_{2}\right\} ; \\
& t_{2}=\operatorname{minimum}\left\{n_{1} n_{2}, n_{1} p_{2}, p_{1} n_{2}, p_{1} p_{2}\right\} ; \\
& t_{3}=\operatorname{maximum}\left\{n_{1} n_{2}, n_{1} p_{2}, p_{1} n_{2}, p_{1} p 2\right\} \text { and }
\end{aligned}
$$


$t_{4}=\operatorname{maximum}\left\{m_{1} m_{2}, m_{1} q_{2}, q_{1} m_{2}, q_{1} q_{2}\right\}$.

\section{Roubast Ranking Technique}

Roubast ranking is a technique which satisfies compensation, linearity, and additive properties and provides results which relates to human intuition. If $\tilde{a}$ is a fuzzy number then the Roubast Ranking is defined by

$$
\boldsymbol{R}(\tilde{a})=\int_{0}^{1} 0.5\left(a_{\alpha}^{L} a_{\alpha}^{U}\right) d \alpha
$$

where $\left\langle a_{\alpha}^{L} a_{\alpha}^{U}\right\rangle$ is the $\alpha$ level cut of the fuzzy number ã and

$$
\left\langle a_{\alpha}^{L} a_{\alpha}^{U}\right\rangle=\{((n-m) \alpha+m),(q-(q-p) \alpha)\}
$$

In this paper we use this method for ranking the objective values. The Roubast ranking index $R$ (ã) gives the representative value of fuzzy number ã.

\section{ALGORITHMS}

The next two sub sections demonstrate the proposed algorithm and an algorithm using traditional approach respectively. These algorithms are illustrated by the authors with the help of a numerical example for a comparative study.

\section{Proposed Algorithm for Modified Vogel Approximation Method}

Step 1: Balance the given transportation problem if not balanced by introducing

A dummy source with fuzzy availability (maximum $\left\{0,\left(m^{\prime}-p^{\prime}\right)-(m-p)\right\}+\operatorname{maximum}\left\{0,\left(p^{\prime}-p\right)\right\}, \operatorname{maximum}\left\{0,\left(m^{\prime}-\right.\right.$ $\left.\left.\left.n^{\prime}\right)-(m-n)\right\}+\operatorname{maximum}\left\{0,\left(p^{\prime}-p\right)\right\}+\operatorname{maximum}\left\{0,\left(n^{\prime}-m^{\prime}\right)-(n-m)\right\}, \operatorname{maximum}\left\{0,\left(p^{\prime}-p\right)\right\}, \operatorname{maximum}\left\{0,\left(q^{\prime}-q\right)\right\}\right)$

And a dummy destination with fuzzy demand (maximum $\left\{0,(m-p)-\left(m^{\prime}-p^{\prime}\right)\right\}+\operatorname{maximum}\left\{0,\left(p-p^{\prime}\right)\right\}, \operatorname{maximum}\{0,(m-$ $\left.\left.p)-\left(m^{\prime}-p^{\prime}\right)\right\}+\operatorname{maximum}\left\{0,\left(p-p^{\prime}\right)\right\}+\operatorname{maximum}\left\{0,(n-m)-\left(n^{\prime}-m^{\prime}\right)\right\}, \quad \operatorname{maximum}\left\{0,\left(p-p^{\prime}\right)\right\}, \operatorname{maximum}\left\{0,\left(q-q^{\prime}\right)\right\}\right)$.

Step 2: Obtain the TOC matrix. (The TOC matrix is obtained by adding the "row opportunity cost matrix" (row opportunity cost matrix: for each row, the smallest cost of that row is subtracted from each element of the same row) and the "column opportunity cost matrix" (Column opportunity cost matrix: for each column of the original transportation cost matrix the smallest cost of that column is subtracted from each element of the same column))

Step 3: Determine the penalty cost for each row and column by subtracting the lowest cell cost in the row or column from the next lowest cell cost in the same row or column.

Step 4: Select the rows or columns with the highest three penalty costs (breaking ties arbitrarily or choosing the lowestcost cell).

Step 5: Compute three transportation costs for selected three rows or columns in step 4 by allocating as much as possible to the feasible cell with the lowest transportation cost.

Step 6: Select minimum transportation cost of three allocations in step 5 (breaking ties arbitrarily or choosing the lowestcost cell).

Step 7: Repeat steps 3-6 until all requirements have been meet.

Step 8: Compute total transportation cost for the feasible allocations using the original unbalanced-transportation cost matrix.

Step 9: Finally obtain the optimal transportation cost for the unbalanced transportation cost matrix using MODI Method.

\section{Important Remarks}

The algorithm will be improved if we add the following two additional steps for breaking ties

(i) If there is a tie in penalty or minimum transportation cost, choose the largest penalty for allocation.

(ii) If there is a tie in penalty and minimum transportation cost, then calculate their corresponding row opportunity cost value/column opportunity cost value, and select the one with maximum.

\section{Algorithm for Simple Vogel Approximation Method}

Step 1: Balance the given transportation problem if not balanced by introducing

a dummy source if total demand $>$ total supply

Or, a dummy destination if total supply $>$ total demand

Step 2: Determine the penalty cost for each row and column by subtracting the lowest cell cost in the row or column from the next lowest cell cost in the same row or column. 
I SSN 2277-3061

Volume: $15 \mathrm{Number}: 6$

International Journal of Computers \& Technology

Step 3: Select the rows or columns with the highest penalty costs (breaking ties arbitrarily or choosing the lowest-cost cell).

Step 4: Allocate as much as possible to the feasible cell with the lowest transportation cost in the row or column with highest penalty cost.

Step 5: Repeat Steps 2, 3, 4 until the entire requirements have been met.

Step 6: Compute total transportation costs for the feasible allocations.

Step 7: Finally obtain the optimal transportation cost for the unbalanced transportation cost matrix using MODI Method.

\section{NUMERICAL EXAMPLE}

Consider the following fuzzy transportation problem given below. All the data in this problem is represented by trapezoidal fuzzy numbers. The problem is solved by the method proposed by the authors of this paper and also by using the traditional approach for comparison. Table 1 represents the numerical problem.

Table 1: Tableau representation of Numerical problem

\begin{tabular}{|l|l|l|l|l|l|}
\hline & 1 & 2 & 3 & 4 & Supply \\
\hline 1 & $(4,6,7,9)$ & $(3,5,7,10)$ & $(5,7,10,12)$ & $(3,4,6,9)$ & $(4,5,7,10)$ \\
\hline 2 & $(2,3,5,9)$ & $(5,7,9,13)$ & $(4,6,9,12)$ & $(5,6,7,10)$ & $(2,3,5,7)$ \\
\hline 3 & $(7,9,10,12)$ & $(6,7,9,10)$ & $(7,9,10,13)$ & $(6,7,10,13)$ & $(7,10,13,14)$ \\
\hline 4 & $(4,5,7,9)$ & $(5,7,12,15)$ & $(7,9,13,15)$ & $(2,4,10,13)$ & $(5,7,10,14)$ \\
\hline Demand & $(4,10,13,15)$ & $(3,7,9,13)$ & $(2,3,10,14)$ & $(3,7,10,13)$ & \\
\hline
\end{tabular}

As, this is unbalanced fuzzy transportation problem, so, to make it balanced fuzzy transportation problem we introduce a Dummy fuzzy column and dummy fuzzy row using Step 1 of algorithm as shown in Table 2.

Table 2: Tableau representation of Balanced Transportation Problem

\begin{tabular}{|l|l|l|l|l|l|l|}
\hline & 1 & 2 & 3 & 4 & 5 & Supply \\
\hline 1 & $(4,6,7,9)$ & $(3,5,7,10)$ & $(5,7,10,12)$ & $(3,4,6,9)$ & $(0,0,0,0)$ & $(4,5,7,10)$ \\
\hline 2 & $(2,3,5,9)$ & $(5,7,9,13)$ & $(4,6,9,12)$ & $(5,6,7,10)$ & $(0,0,0,0)$ & $(2,3,5,7)$ \\
\hline 3 & $(7,9,10,12)$ & $(6,7,9,10)$ & $(7,9,10,13)$ & $(6,7,10,13)$ & $(0,0,0,0)$ & $(7,10,13,14)$ \\
\hline 4 & $(4,5,7,9)$ & $(5,7,12,15)$ & $(7,9,13,15)$ & $(2,4,10,13)$ & $(0,0,0,0)$ & $(5,7,10,14)$ \\
\hline 5 & $(0,0,0,0)$ & $(0,0,0,0)$ & $(0,0,0,0)$ & $(0,0,0,0)$ & $(0,0,0,0)$ & $(7,5,7,10)$ \\
\hline Demand & $(4,10,13,15)$ & $(3,7,9,13)$ & $(2,3,10,14)$ & $(3,7,10,13)$ & $(13,13,0,0)$ & $(25,40,42,55)$ \\
\hline
\end{tabular}

Now, Roubast Ranking Technique for the numerical problem is as shown in the Table 3 shown below.

Table 3: Ranking of all fuzzy supply points and fuzzy demand points

\begin{tabular}{|l|l|}
\hline$\tilde{A}_{11}(4,6,7,9)$ & $R\left(\tilde{A}_{11}\right)=\int_{0}^{1} 0.5[((6-4) \alpha+4)+(9+(9-7) \alpha)] \mathrm{d} \alpha=6.5$ \\
\hline$\tilde{A}_{12}(3,5,7,10)$ & $R\left(\tilde{A}_{12}\right)=\int_{0}^{1} 0.5[((5-3) \alpha+3)+(10-(10-7) \alpha)] \mathrm{d} \alpha=6.25$ \\
\hline$\tilde{A}_{13}(5,7,10,12)$ & $R\left(\tilde{A}_{13}\right)=\int_{0}^{1} 0.5[((7-5) \alpha+5)+(12-(12-10) \alpha)] \mathrm{d} \alpha=8.5$ \\
\hline$\tilde{A}_{14}(3,4,6,9)$ & $R\left(\tilde{A}_{14}\right)=\int_{0}^{1} 0.5[((4-3) \alpha+3)+(9-(9-6) \alpha)] \mathrm{d} \alpha=5.75$ \\
\hline$\tilde{A}_{15}(0,0,0,0)$ & $R\left(\tilde{A}_{15}\right)=0$ \\
\hline
\end{tabular}




\begin{tabular}{|c|c|}
\hline$\widetilde{A_{21}}(2,3,5,9)$ & $R\left(\tilde{A}_{21}\right)=\int_{0}^{1} 0.5[((3-2) \alpha+2)+(9-(9-5) \alpha)] \mathrm{d} \alpha=4.75$ \\
\hline$\tilde{A}_{22}(5,7,9,13)$ & $R\left(\tilde{A}_{22}\right)=\int_{0}^{1} 0.5[((7-5) \alpha+5)+(13-(13-9) \alpha)] \mathrm{d} \alpha=8.5$ \\
\hline$\tilde{A}_{23}(4,6,9,12)$ & $12-(12-9) \alpha)] d \alpha=7.75$ \\
\hline$\tilde{A}_{24}(5,6,7,10)$ & $R\left(\tilde{A}_{24}\right)=\int_{0}^{1} 0.5[((6-5) \alpha+5)+(10-(10-7) \alpha)] d \alpha=7$ \\
\hline$\tilde{A}_{25}(0,0,0,0)$ & $\boldsymbol{R}\left(\tilde{A}_{25}\right)=0$ \\
\hline$\widetilde{A}_{31}(7,9,10,12)$ & $R\left(\tilde{A}_{31}\right)=\int_{0}^{1} 0.5[((9-7) \alpha+7)+$ \\
\hline$\tilde{A}_{32}(6,7,9,10)$ & $10-(10-9) \alpha)] \mathrm{d} \alpha=8$ \\
\hline$\tilde{A}_{33}(7,9,10,13)$ & $13-(13-10) \alpha)] \mathrm{d} \alpha=9.75$ \\
\hline$\tilde{A}_{34}(6,7,10,13)$ & $(13-(13-10) \alpha)]_{\mathrm{da}=9}$ \\
\hline$\widetilde{A}_{35}(0,0,0,0)$ & $R\left(\tilde{A}_{35}\right)=0$ \\
\hline$\widetilde{A}_{41}(4,5,7,9)$ & $-(9-7) \alpha)] d \alpha=6.25$ \\
\hline$\widetilde{A}_{42}(5,7,12,15)$ & $R\left(\tilde{A}_{42}\right)=\int_{0}^{1} 0.5[((7-5) \alpha+5)+(15-(15-12) \alpha)] \mathrm{d} \alpha=9.75$ \\
\hline$\widetilde{A}_{43}(7,9,13,15)$ & $9-7) \alpha+7)+(15-(15-13) \alpha)]_{\mathrm{da}=11}$ \\
\hline$\widetilde{A}_{44}(2,4,10,13)$ & $(4-2) \alpha+2)+(13-(13-10) \alpha)] \mathrm{d} \alpha=7.25$ \\
\hline$\widetilde{A}_{45}(0,0,0,0)$ & $R\left(\widetilde{A_{45}}\right)=0$ \\
\hline$\tilde{A}_{51}(0,0,0,0)$ & $R\left(\widetilde{A_{51}}\right)=0$ \\
\hline$\widetilde{A_{52}}(0,0,0,0)$ & $R\left(\tilde{A}_{52}\right)=0$ \\
\hline$\widetilde{A}_{53}(0,0,0,0)$ & $R\left(\widetilde{A}_{53}\right)=0$ \\
\hline$\widetilde{A}_{54}(0,0,0,0)$ & $R\left(\widetilde{A}_{54}\right)=0$ \\
\hline$\widetilde{A}_{55}(0,0,0,0)$ & $R\left(\tilde{A}_{55}\right)=0$ \\
\hline
\end{tabular}

Fuzzy Supplies are

Table 4: Ranking of fuzzy Supplies

\begin{tabular}{|l|l|}
\hline$\tilde{a}_{1}(4,5,7,10)$ & $R\left(\tilde{a}_{1}\right)=\int_{0}^{1} 0.5[((5-4) \alpha+4)+(10-(10-7) \alpha)] \mathrm{d} \alpha=6.5$ \\
\hline$\tilde{a}_{2}(2,3,5,7)$ & $R\left(\tilde{a}_{2}\right)=\int_{0}^{1} 0.5[((3-2) \alpha+2)+(7-(7-5) \alpha)] \mathrm{da}=4.25$ \\
\hline
\end{tabular}




\begin{tabular}{|l|l|}
\hline$\tilde{a}_{3}(7,10,13,14)$ & $R\left(\tilde{a}_{3}\right)=\int_{0}^{1} 0.5[((10-7) \alpha+7)+(14-(14-13) \alpha)] \mathrm{da}=11$ \\
\hline$\tilde{a}_{4}(5,7,10,14)$ & $R\left(\tilde{a}_{4}\right)=\int_{0}^{1} 0.5[((7-5) \alpha+5)+(14-(14-10) \alpha)] \mathrm{da}=9$ \\
\hline$\tilde{a}_{5}(7,15,7,10)$ & $R\left(\tilde{a}_{5}\right)=\int_{0}^{1} 0.5[((15-7) \alpha+7)+(10-(10-7) \alpha)] \mathrm{da}=9.75$ \\
\hline
\end{tabular}

Fuzzy Demands are

Table 5: Ranking of fuzzy Demands

\begin{tabular}{|l|l|}
\hline$\widetilde{b}_{1}(4,10,13,15)$ & $R\left(\widetilde{b}_{1}\right)=\int_{0}^{1} 0.5[((10-4) \alpha+4)+(15-(15-13) \alpha)] \mathrm{da}=10.5$ \\
\hline$\widetilde{b}_{2}(3,7,9,13)$ & $R\left(\widetilde{b}_{2}\right)=\int_{0}^{1} 0.5[((7-3) \alpha+3)+(13-(13-9) \alpha)] \mathrm{d} \alpha=8$ \\
\hline$\widetilde{b}_{3}(2,3,10,14)$ & $R\left(\widetilde{b}_{3}\right)=\int_{0}^{1} 0.5[((3-2) \alpha+2)+(14-(14-10) \alpha)] \mathrm{d} \alpha=7.25$ \\
\hline$\widetilde{b}_{4}(3,7,10,13)$ & $R\left(\tilde{b}_{4}\right)=\int_{0}^{1} 0.5[((7-3) \alpha+3)+(13-(13-10) \alpha)] \mathrm{d} \alpha=8.25$ \\
\hline$\widetilde{b}_{5}(13,13,0,0)$ & $R\left(\widetilde{b}_{5}\right)=\int_{0}^{1} 0.5[((13-13) \alpha+13)+(0-(0-0) \alpha)] \mathrm{d} \alpha=6.5$ \\
\hline
\end{tabular}

Using the various entries from Table 2, 3 and 4 the fuzzy transportation problem is reduced to crisp transportation problem. Table 6 represents crisp transportation problem

Table6: Tableau representation of balanced transportation problem using proposed method and ranking

\begin{tabular}{|l|l|l|l|l|l|l|}
\hline & 1 & 2 & 3 & 4 & 5 & Supply \\
\hline 1 & 6.5 & 6.25 & 8.5 & 5.75 & 0 & 6.5 \\
\hline 2 & 4.75 & 8.5 & 7.75 & 7 & 0 & 4.25 \\
\hline 3 & 9.5 & 8 & 9.75 & 9 & 0 & 11 \\
\hline 4 & 6.25 & 9.75 & 11 & 7.25 & 0 & 9 \\
\hline 5 & 0 & 0 & 0 & 0 & 0 & 9.75 \\
\hline Demand & 10.5 & 8 & 7.25 & 8.25 & 6.5 & 40.5 \\
\hline
\end{tabular}

After

Improved VAM,

$X_{12}=5.5, X_{14}=1, X_{21}=4.25, X_{34}=4.5, X_{35}=6.5, X_{41}=6.25, X_{44}=2.75, X_{52}=2.5, X_{53}=7.25$ and the optimal solution obtained for this unbalanced problem is $X_{0}=153$.

Now, we apply simple VAM algorithm in Table 7.

Table 7: Tableau representation of balanced transportation problem using Simple VAM and Ranking

\begin{tabular}{|l|l|l|l|l|l|}
\hline & 1 & 2 & 3 & 4 & Supply \\
\hline 1 & 6.5 & 6.25 & 8.5 & 5.75 & 6.5 \\
\hline 2 & 4.75 & 8.5 & 7.75 & 7 & 4.25 \\
\hline 3 & 9.5 & 8 & 9.75 & 9 & 11 \\
\hline 4 & 6.25 & 9.75 & 11 & 7.25 & 9 \\
\hline 5 & 0 & 0 & 0 & 0 & 3.25 \\
\hline Demand & 10.5 & 8 & 7.25 & 8.25 & 34 \\
\hline
\end{tabular}


After applying simple VAM,

$X_{12}=1, X_{14}=5.5, X_{21}=4.25, X_{32}=7, X_{33}=4, X_{41}=6.25, X_{44}=2.25, X_{53}=0.25$ and the best feasible solution obtained is 208.4375 . Using this method, we do not obtain any optimal solution.

\section{CONCLUSION}

Using the traditional Simple VAM method, we do not obtain any optimal solution. Also, the best feasible solution obtained using the traditional method gives higher numerical value of transportation cost as compared to the solution obtained using the Modified VAM method, which is optimal as well.

\section{ADVANTAGES}

The proposed method is easy to understand and to apply for obtaining the fuzzy optimal solution of unbalanced fuzzy transportation problems existing in real life situations. This algorithm can also be used for solving various other problems also like, project schedules, assignment problems, network flow problems etc. The result obtained here is better than the result obtained using simple VAM or any other method.

\section{ACKNOWLEDGMENTS}

Our thanks to the experts who have contributed towards development of the manuscript.

\section{REFERENCES}

I. Zadeh, L. A.. 1965. Fuzzy sets. Information and control, 8, 338-353.

II. Chanas, S., Kuchta, D. 1996.A concept of the optimal solution of the transportation problem with fuzzy cost coefficients. Fuzzy Sets and Systems 82, 299-305.

III. Liu, Shiang-Tai., Chiang, Kao. 2004. Solving fuzzy transportation problems based on extension principle, European Journal of Operational Research, 153, 661-674.

IV. Saad, O. M., Abbas, S. A. 2003. A parametric study on transportation problem under fuzzy environment. The Journal of Fuzzy Mathematics, 115-124.

V. Gani, A., Razak, K. A. 2006. Two stage fuzzy transportation problem. Journal of physical sciences, 63-69.

VI. Hitchcock, F. L. 1941. The distribution of a product from several sources to numerous localities. Journal of Math. Phys. 20, 224-230.

VII. Dantzig, G. B. 1963.Linear Programming and Extensions. Princeton University Press, Princeton.

VIII. Chen, S. H. 1985. Operations on fuzzy numbers with function principle. Tamkang Journal of Management Sciences, 6, 13-25.

IX. Riggs, J. L., Inoue, M. S. 1975. Introduction to Operation Research and Management Science. McGraw-Hill, New York.

X. Joshi, N., Chauhan, S. S. 2013. Solution of Fuzzy Transportation Problem using Improved VAM with Roubast Ranking Technique. International Journal of Computer Applications 82(15), 6-8.

XI. Saini, R. K., Sanghal, A., and Prakash, Om. 2015. Unbalanced problems in fuzzy environment using centroid ranking technique. International Journal of Computer Applications, 110(11), 27-33.

XII. Rani, D., Gulati, T. R., and Kumar, A. 2014. A method for unbalanced transportation problems in fuzzy environment. Sadhana, 39(3), 573-581.

XIII. Edward Samuel, A., and Venkatachalapathy, M. 2013. IZPM for unbalanced fuzzy transportation problems. International Journal of Pure and Applied Mathematics, 86(4), 689-700.

XIV. Kadhirvel, K., Balamurugan, K. 2013. Method for solving unbalanced transportation problems using trapezoidal fuzzy numbers. IJERA, 3(4), 2591-2596.

XV. Edward Samuel, A., Venkatachalapathy, M. 2012. A new dual based approach for the unbalanced fuzzy transportation problem. Applied Mathematical Sciences, 6(89), 4443- 4455.

XVI. Sobha, K. R. 2014. Profit maximization of unbalanced fuzzy transportation problem. International Journal of Science and Research, 3(11), 2300-2302.

XVII. Kumar, A., Kaur, A. 2011. Application of linear programming for solving fuzzy transportation problems. J. Appl. Math and Informatics, 29(3-4), 831-846.

XVIII. Kumar, A., Kaur, A. 2012. Methods for solving unbalanced fuzzy transportation problems. Operational Research, 12(3), 287-316.

XIX. Kaur, A., Kumar, A. 2011. A new method for solving fuzzy transportation problems using ranking function. Applied Mathematical Modelling, 35(12), 5652-5661.

XX. Pandian, P., Natarajan, G. 2010. A new algorithm for finding a fuzzy optimal solution for fuzzy transportation problems. Applied Mathematical Sciences, 4, 79-90. 


\section{Author' biography with Photo}

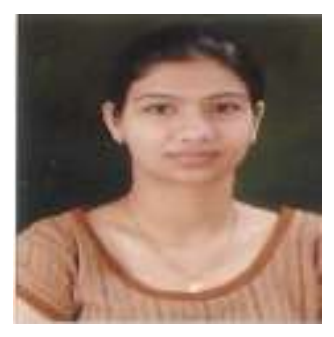

Ms. Nidhi Joshi is a Ph.D research scholar under the supervision of Dr. Surjeet Singh Chauhan (Gonder). She has completed her M.Sc (Mathematics) from Punjab University, Chandigarh, India. Her area of research is Fuzzy Transportation.

Punjab Technical University, Jalandhar (Punjab), India.

e-mail: nids_22@yahoo.co.in

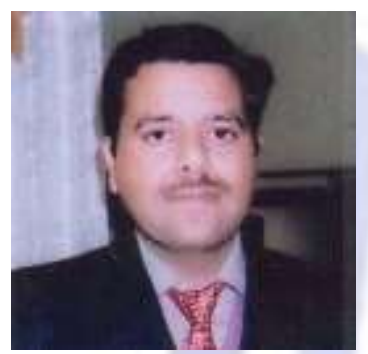

Dr. Surjeet Singh Chauhan(Gonder) (Prof.) is Head of Department in Applied Sciences Department, Chandigarh Group of Colleges, Gharuan, Mohali (Punjab), India. His current area of research is Fuzzy Optimization.

Applied Sciences Department, Chandigarh Group of Colleges, Gharuan, Mohali (Punjab),India.

e-mail: surjeetschauhan@yahoo.com 